\title{
Hearing in action; auditory properties of neurons in the red nucleus of alert primates
}

\author{
Jonathan M. Lovell ${ }^{1,2}{ }^{*}$, Judith Mylius ${ }^{1}$, Henning Scheich ${ }^{1}$ and Michael Brosch ${ }^{1}$ \\ 1 Special Lab for Primate Neurobiology, Leibniz Institute for Neurobiology, Magdeburg, Germany \\ 2 Deutsches Zentrum für Neurodegenerative Erkrankungen, Magdeburg, Germany
}

\author{
Edited by: \\ Monica Munoz-Lopez, University of \\ Castilla-La Mancha, Spain \\ Reviewed by: \\ Simon Baumann, Newcastle \\ University, UK \\ Manuel S. Malmierca, University of \\ Salamanca, Spain \\ *Correspondence: \\ Jonathan M. Lovell, Special Lab for \\ Primate Neurobiology, Leibniz \\ Institute for Neurobiology, \\ Brenneckestrasse 6, \\ 39118 Magdeburg, Germany \\ e-mail: jlovell@ifn-magdeburg.de
}

\begin{abstract}
The response of neurons in the Red Nucleus pars magnocellularis (RNm) to both tone bursts and electrical stimulation were observed in three cynomolgus monkeys (Macaca fascicularis), in a series of studies primarily designed to characterize the influence of the dopaminergic ventral midbrain on auditory processing. Compared to its role in motor behavior, little is known about the sensory response properties of neurons in the red nucleus (RN); particularly those concerning the auditory modality. Sites in the RN were recognized by observing electrically evoked body movements characteristic for this deep brain structure. In this study we applied brief monopolar electrical stimulation to 118 deep brain sites at a maximum intensity of $200 \mu \mathrm{A}$, thus evoking minimal body movements. Auditory sensitivity of RN neurons was analyzed more thoroughly at 15 sites, with the majority exhibiting broad tuning curves and phase locking up to $1.03 \mathrm{kHz}$. Since the RN appears to receive inputs from a very early stage of the ascending auditory system, our results suggest that sounds can modify the motor control exerted by this brain nucleus. At selected locations, we also tested for the presence of functional connections between the $\mathrm{RN}$ and the auditory cortex by inserting additional microelectrodes into the auditory cortex and investigating how action potentials and local field potentials (LFPs) were affected by electrical stimulation of the RN.
\end{abstract}

Keywords: red nucleus, auditory, primate, electrophysiology, neuron

\section{INTRODUCTION}

The Red Nucleus (RN) or nucleus ruber lies in the rostral midbrain and derives its name from the high concentration of ironcontaining pigments within its cellular structure (Hernandez, 1931). The RN is comprised of two subnuclei, the rostral pars parvocellularis (Oka and Jinnai, 1978; Onodera and Hicks, 2009), and the smaller caudal pars magnocellularis (Yamaguchi and Goto, 2006). Gibson et al. (1985) found that in rhesus monkeys, the magnocellular RN controls the onset, velocity, and duration of specific upper limb movements, with electrical stimulation causing discrete contractions of limb muscles in the shoulder, elbow, wrist, and digits.

Compared to its role in motor behavior little is known about sensory response properties of neurons in the RN, particularly those concerning the auditory modality. In chloraloseanesthetized cats, neurons in the rostral RN have been found to exhibit short-latency responses to clicks and tones (Massion and Albe-Fessard, 1963; Irvine, 1980). Tuning curves are generally very broad, and neurons receive inputs from both ears and are sensitive to interaural time and level differences (Shinkarenko, 1984; Shinkarenko et al., 1985). Bratus et al. (1981) recorded evoked potentials from the magnocellular $\mathrm{RN}$ in anesthetized cats, and showed that the response latency varies from 3.5 to $10.5 \mathrm{~ms}$, depending on the intensity of the auditory stimulation. It has been hypothesized that the $\mathrm{RN}$ is a component of the subcortical path for reflexes connected with turning the ear in the direction of sound (Courville, 1968), and that it is a part of the complex pathways concerned with the animal's postural and defensive responses to acoustic stimulation (Martin and Dom, 1970).

Here we report auditory response properties of the $\mathrm{RN}$ in alert macaque monkeys. These data were obtained in a series of studies that were primarily designed to characterize effects of the dopaminergic ventral midbrain on auditory processing. The auditory responses in the RN attracted our attention because of their potential role in audiomotor interactions, i.e., its possible involvement in guiding movement-related reactions to sounds, and its involvement in monitoring the sounds that are evoked by movement.

In these experiments, we moved microelectrodes along tracks oriented approximately in a mediolateral direction, through different deep brain structures toward RN. Sites in the RN were recognized by observing electrically evoked body movements characteristic for this deep brain structure. At the same time, the electrodes served to record action potentials and local field potentials (LFPs) from the RN, and to characterize neuronal responses to clicks and pure tones. At selected locations, we also tested for the presence of functional connections between the RN and the auditory cortex by inserting additional microelectrodes into the auditory cortex and analysing how action potentials and LFPs were affected by electrical stimulation of the RN. 


\section{MATERIALS AND METHODS}

The subjects used in this experiment were three adult cynomolgus monkeys (Macaca fascicularis); monkey E (male, $5.5 \mathrm{~kg}$ ) monkey $\mathrm{W}$ (male, $6.2 \mathrm{~kg}$ ), and monkey M (female, $4 \mathrm{~kg}$ ). Each monkey was fitted with a head holder and a recording chamber $(18 \mathrm{~mm}$ diameter), which was positioned in the right (monkeys $\mathrm{E}$ and $\mathrm{M}$ ) and the left (monkey W) temporal regions of the skull. Details of the surgery are given elsewhere (Brosch and Scheich, 2008). Experiments were conducted in a sound-attenuated doublewalled room. During the experiments the monkeys were seated in a primate chair and their heads were fixed to allow for acute recordings. All experiments were carried out under approval of the animal care and ethics committee of the State Sachsen-Anhalt (No. 28-14 42502/2-806 IfN) and in accordance with the guidelines for animal experimentation of the European Communities Council Directive (86/609/EEC).

Micro-fiber electrodes were used for both electrical stimulation and recording neuronal activity. The electrodes were constructed from a single fiber with a $25 \mu \mathrm{m}$ tungsten and iridium core, insulated with quartz glass to a total diameter of $80 \mu \mathrm{m}$, with tips sharpened to a point using an ultrafine diamond grinder. The impedance of each electrode was tested and found to be in the ranges of $0.5 \mathrm{M} \Omega$ for recording electrodes to less than $25 \mathrm{k} \Omega$ for low impedance stimulation electrodes. The electrodes were then fashioned to fit in the micro-drive and were able to extend over $40 \mathrm{~mm}$ through the macaque brain. Electrodes were advanced remotely into the brain from outside of the sound-attenuating room using a seven-channel microdrive (System Eckhorn, Thomas Recording).

Through these electrodes, we simultaneously recorded multiunit activity using a band-pass filter set between 1 to $7 \mathrm{kHz}$, and
LFPs with a band-pass of 0.1 to $250 \mathrm{~Hz}$, using the systems SUA-02 and LFP-03 (Thomas Recording). All signals were fed into an AD data-acquisition system (32-channel Alpha-Map, Alpha-Omega). The LFP sampling rate was $658 \mathrm{~Hz}$, and for multiunit activity it was $50 \mathrm{kHz}$. We only stored time stamps of multiunit activity when spike amplitudes exceeded noise thresholds.

Initially, MRI images were used to plan electrode trajectories toward the RN. As can be seen in Figure 1A, the iron rich pigmentation within the $\mathrm{RN}$ makes this structure highly visible when imaged using MRI. The temporal location of the recording chambers restricted the electrode trajectories to those shown in Figure 1B Thus, electrodes were inserted into the brain at an angle of $18( \pm 6)$ degrees from the horizontal plane. Once the electrodes had been advanced to around $19 \mathrm{~mm}$ from the surface of the dura membrane, we commenced presentation of electrical and auditory stimulation to the monkey. Finer localization of the two red nuclei was pinpointed by observing upper body movements (e.g., arms, shoulders, face, mouth, and eyelids) in response to the electrical stimulation (note that the monkeys were head fixed and seated in a primate chair, thus limiting the full expression of certain electrically evoked movements). No histological verification of stimulation sites was undertaken because more than one hundred stimulation sites were tested in each monkey and because all monkeys in the current study are still involved in other research projects, following the principles of Replacement, Reduction, and Refinement in animal experimentation.

Multiunit activity and LFPs were recorded from the auditory cortex using a 16-channel microdrive (System Eckhorn, Thomas Recording). The microdrive was oriented slightly off the dorsoventral plane $\left(10^{\circ}\right)$. Electrodes entered the cortex between
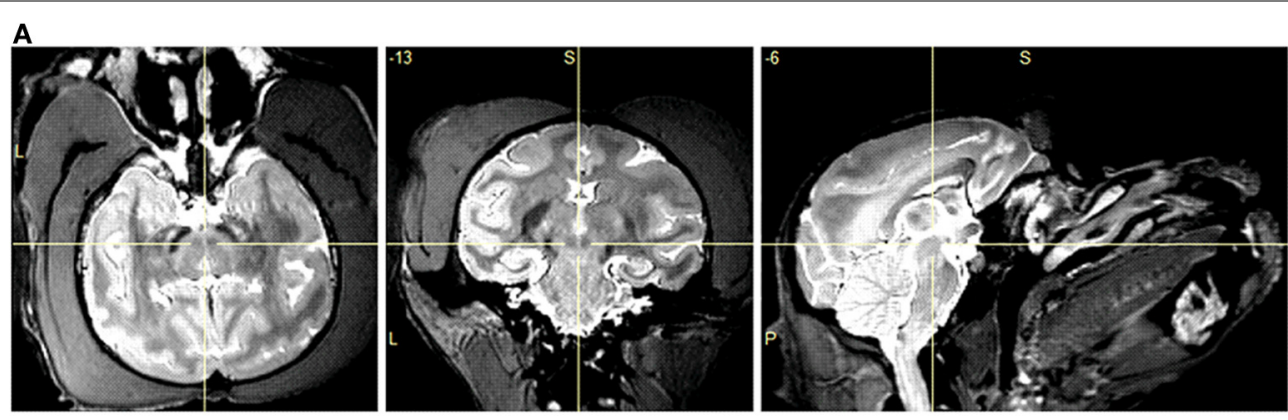

B

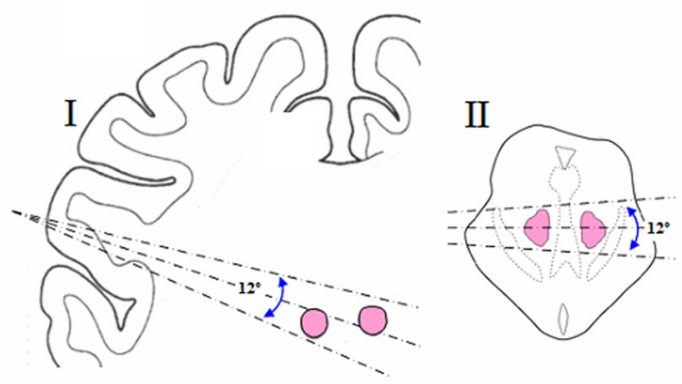

FIGURE 1 | (A) MRI images of the macaque brain (monkey E) in axial, coronal, and sagittal views, with the cross hair showing the location of the red nucleus. (B) Range of electrode angles used in the sagittal (I) and axial (II) planes. 
stereotactic coordinates A5 to A9 and D7.5 to D12.5 and subsequently were moved through the parietal cortex into the core fields of the auditory cortex.

Monopolar electrical stimulation was delivered using a Multichannel Systems STG 400-4 stimulator, programmed to generate $50 \mathrm{~ms}$ trains of square edged biphasic pulses. These were presented at a frequency of $100 \mathrm{~Hz}$, and a pulse width of $700 \mu \mathrm{s}$ and at a maximum intensity of $200 \mu \mathrm{A}$, to evoke minimal body movements only. We presented acoustic stimulation in the form of clicks and tone bursts. Clicks were produced by a waveform generator (WG1, Tucker-Davis Technologies) and presented every $2 \mathrm{~s}$ during each of the electrode tracks. Tone bursts were generated using a computer coupled to an array processor (Tucker-Davis Technologies, Gainesville, FL), which converted the signal from digital to analog at a sample rate of $100 \mathrm{kHz}$. Each frequency (40 in total) was repeated 10 times, in pseudorandom order, from
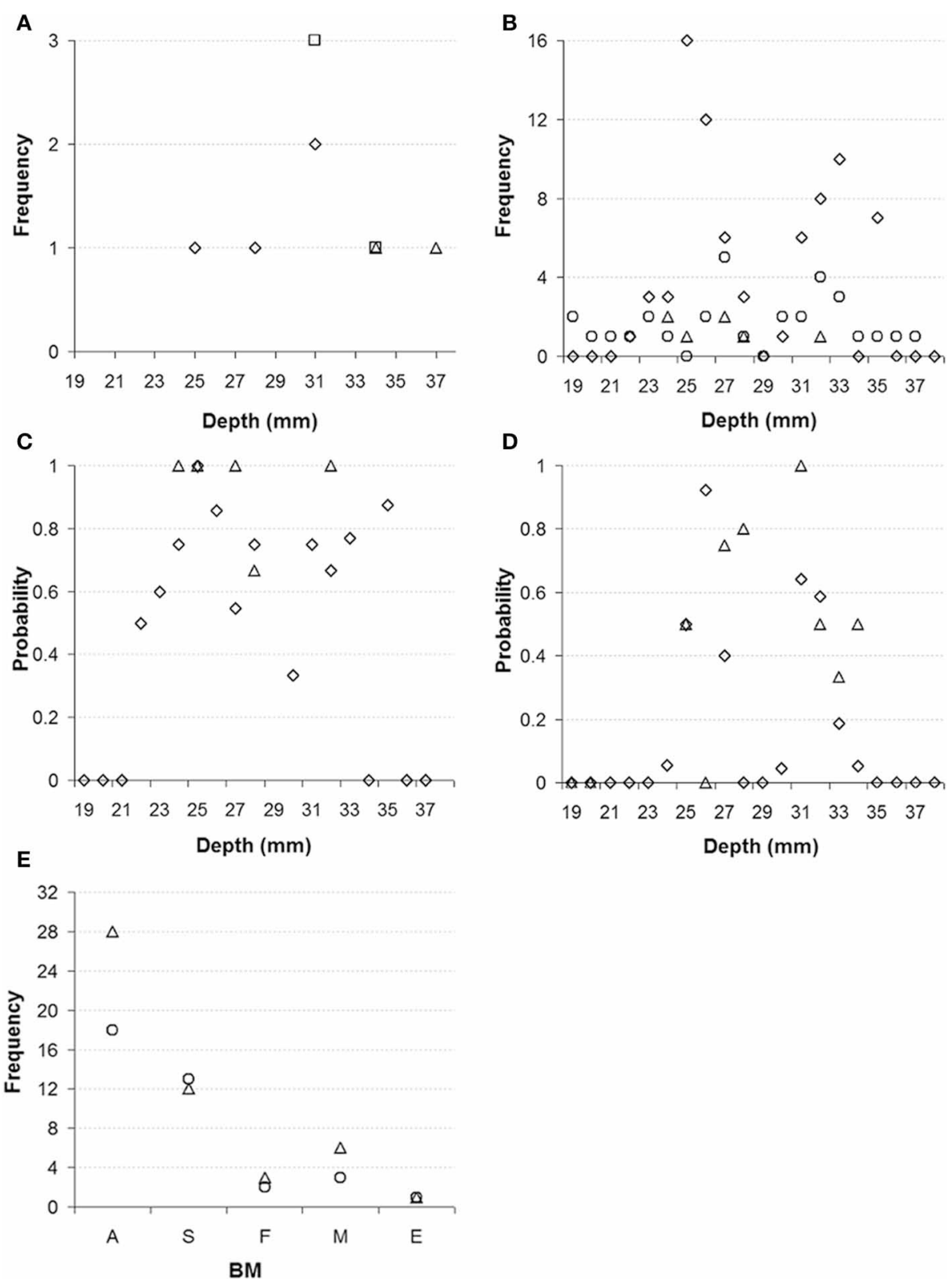

FIGURE 2 | (A) Representative electrode track through the left and right red nucleus in monkey E. Diamonds represent where electrical stimulation evoked upper body movements (indicative of red nucleus), squares represent responses to auditory stimulation, and triangles represent locations where no responses to electrical stimulation were observed. (B) Frequency of sites where electrical stimulation evoked upper body movement for all electrode tracks (diamonds monkey $\mathrm{E}$, triangles monkey $\mathrm{W}$ ) and frequency of

unresponsive sites (circles). (C) Probability of sites with electrical evoked upper body movements (diamonds monkey E, triangles monkey W). (D) Probability of sites with auditory responses (diamonds monkey $E$, triangles monkey W). (E) Category and frequency of the main body movements evoked by the electrical stimulation of the left and right $\mathrm{RN}$ (monkeys $\mathrm{E}$ and W combined). A, arm; $S$, shoulder; F, face; $M$, mouth; $E$, eye (left nucleus: circles, right nucleus: triangles). 
0.11 to $27.2 \mathrm{kHz}$, covering a range of 8 octaves spaced by 0.2 of an octave. The tone burst envelope was $100 \mathrm{~ms}$ in duration, ramped by $5 \mathrm{~ms}$ with an inter tone interval of $900 \mathrm{~ms}$. The auditory stimulus gain was increased using an amplifier (model A202; Pioneer, Long Beach, CA), then fed to two bilaterally placed free-field loudspeakers (Canton Karat 720.2), positioned $1.1 \mathrm{~m}$ from the subject, at intensities not exceeding $65 \mathrm{~dB}$ (re. $20 \mu \mathrm{Pa}$ ). The sound pressure level was measured with a free-field $1 / 2^{\prime}$ microphone (40AC, G.R.A.S.), located near to the monkey's head.

Custom written MATLAB (version 2007b, MathWorks, Natick, MA, USA) programs were used for the off-line analyses of multiunit and LFP recordings. In the $\mathrm{RN}$, tuning curves were generated from each multiunit recording of responses to the tone bursts which were presented at 40 different frequencies, as described elsewhere (Brosch et al., 1999). From these tuning curves, we obtained the best frequency, the bandwidth, the firstspike latency, and the last-spike latency. In the auditory cortex, we computed a post-stimulus time histogram (PSTH) with a bin size of $10 \mathrm{~ms}$ for each of the multiunit recordings, relative to the onset of electrical stimulation in the $\mathrm{RN}$ ( $\geq 50$ trials). Because the electrical stimulation generated an artifact that lasted maximally $20 \mathrm{~ms}$, this period was excluded from the analysis. A multiunit recording was considered to have responded to the electrical stimulation if the number of discharges within at least two of 30 consecutive 10 -ms post-stimulus bins was significantly above the number of discharges immediately before stimulus presentation (Wilcoxon signed rank tests, two-sided). For each of the LFP recordings, evoked potentials were calculated by averaging the LFP relative to the onset of auditory or electrical stimulation. In order to analyse auditory-evoked potentials, the lowest value of the first main trough of the waveform was used to indicate the response latency and frequency bandwidth.

\section{RESULTS}

In this study we applied brief electrical stimulation to 118 deep brain sites in two monkeys (monkey E and monkey W). Figure 2A shows the results of a representative track through the red nuclei, following the center dashed line from Figure 1B. At 86 of these sites (76 in monkey E and 10 in monkey W) we could evoke upper body movements that are characteristic for the RN. Figures 2B,C show the frequency and probability of evoked upper body movements and no responses to the electrical stimulation for all tracks in monkeys $\mathrm{E}$ and $\mathrm{W}$. At the remaining sites, no such movements were observed. Upper body and face movements were exclusively observed in two clusters between 22 and $28 \mathrm{~mm}$ and between 30 and $35 \mathrm{~mm}$ from the dura surface, which is in good correspondence to the location of the two red nuclei in both hemispheres and relative to published brain atlases (see Figure 1B).

When we recorded action potentials from these 118 stimulation sites, we found that 18 sites ( 14 in monkey $\mathrm{E}$ and 4 in monkey $\mathrm{W}$ ) responded to the click stimulation, which was presented every 2 s to the monkeys. With one exception, the auditory responses were found exclusively at the 86 sites where electrical stimulation evoked upper body movements. Thus, auditory responses were found at $19.7 \%$ of the sites in the RN. We also tested the click stimulation at another 335 sites (312 in monkey
E and 23 in monkey W) at which electrical stimulation was not applied directly to reduce the number of electrical stimulations delivered to the monkeys, but which fell within $200 \mu \mathrm{m}$ from a site from which upper body and face movements were elicited. Of these sites, most are presumed to be located in the RN; 67 sites (19.5\%; 55 in monkey E and 12 in monkey W) did respond to the click stimulation. Figure $2 \mathrm{D}$ shows the probability for finding click responses for all of the 335 sites in and around the RN. Figure 2E shows the category and frequency of the main body movements evoked by electrical stimulation of the left and right RN.

Indication for auditory responses in the RN was also obtained at 23 of 51 sites in a third monkey (monkey M). From this monkey, neuronal recordings were obtained from a brain region that corresponded to that tested in monkeys E and W, and following the same stereotactic coordinates used on the two monkeys. Electrical stimulation was omitted so long as the electrode was estimated to be within the $\mathrm{RN}$, in order to reserve electrical
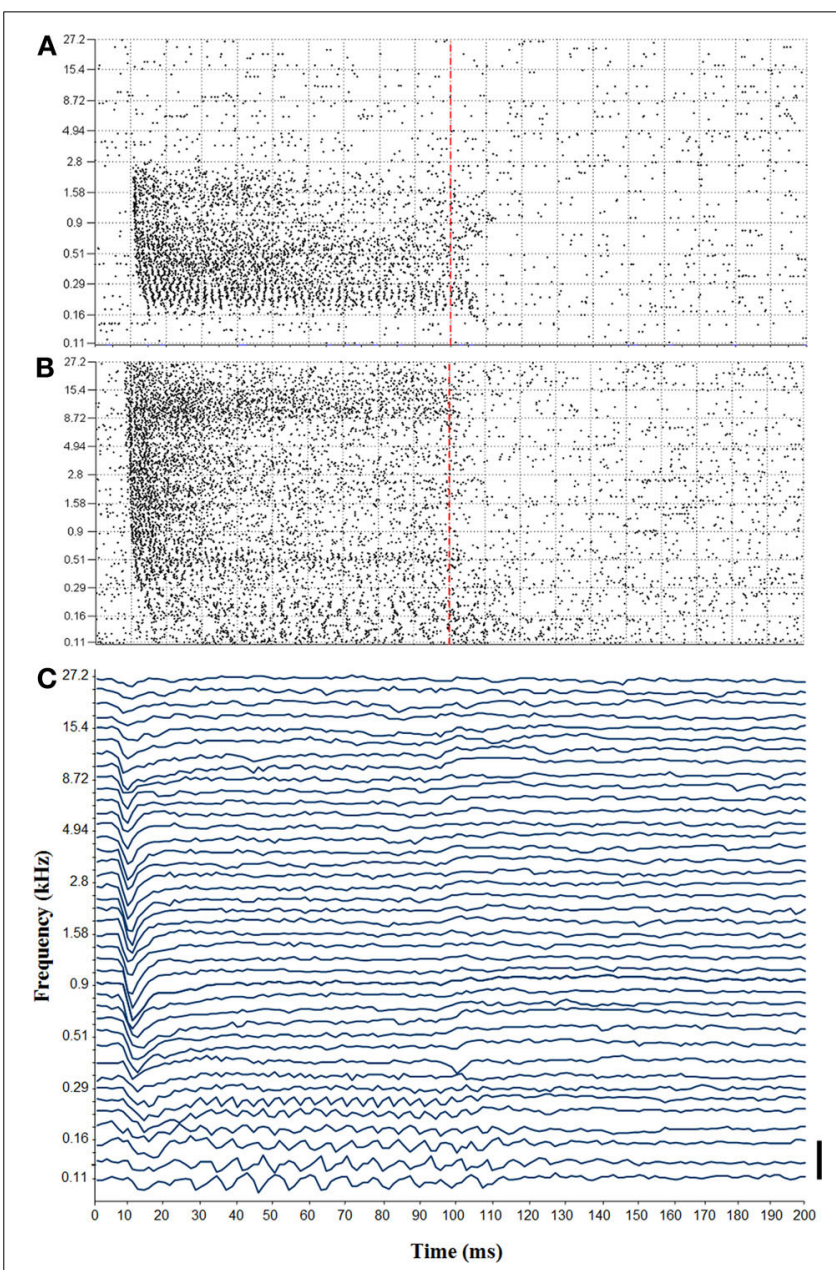

FIGURE 3 | (A) Tuning curve recorded from the left red nucleus. Vertical dashed line indicates the end of the tone bursts. (B) Tuning curve from the right red nucleus. Note the temporal coding showing phase locking to low frequency tones. (C) Auditory-evoked potential recorded in responses to tone bursts from the right red nucleus (bar $=100 \mu \mathrm{V}$ ). 
stimulation exclusively for locations within the ventral tegmental area where the monkey would receive brain stimulation reward for bar pressing. In this experiment, the presence of auditory responses around the estimated depth of the RN was actually used as a landmark to determine the position of the proximal ventral tegmental area.

Auditory sensitivity of RN neurons was analyzed more thoroughly at 15 sites ( 12 in monkey $\mathrm{E}$ and 3 in monkey $\mathrm{W})$, where strong auditory responses to clicks were observed and from where upper body movements could be electrically evoked, or which fell within $200 \mu \mathrm{m}$ from such a site. To achieve this, we presented 400 tone bursts over a range of frequencies from 0.11 to $27.2 \mathrm{kHz}$. Figures $\mathbf{3 A}, \mathbf{B}$ shows spike responses to the tonal stimuli from two representative sites.

The auditory sensitivity of the RN was also detected in the LFPs that were recorded in parallel with the spikes. Figure $3 \mathrm{C}$ shows auditory-evoked potentials that were obtained from the same site at which the tuning curve was obtained from the spike responses shown in Figure 3B. We noticed that, similar to the spike responses, auditory-evoked potentials were phase locked to low stimulus frequencies during the full duration of the tones (note that our filter setting did not allow for us to reliably detect frequency following responses above $250 \mathrm{~Hz}$ ). For higher stimulus frequencies, by contrast, auditory-evoked potentials consisted only of an initial, negative going wave that occurred about $10 \mathrm{~ms}$
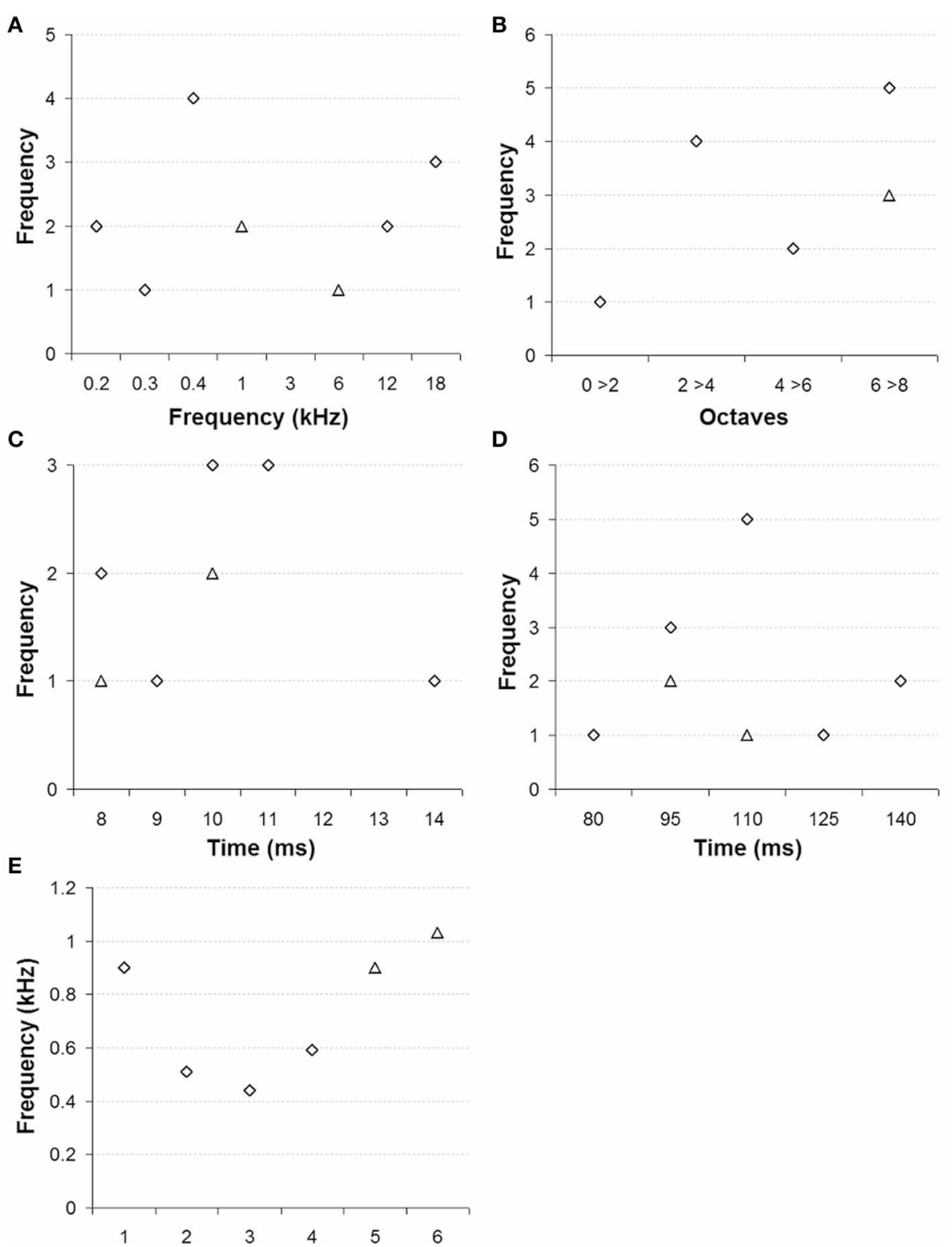

FIGURE 4 | Tonal selectivity of multiunit activity in red nucleus. (A) Best frequency, (B) Tuning curve bandwidths in octaves, (C) Latency of the first spike, (D) Latency of the last spike, and (E) Maximum frequency of phase locking (monkey E: diamonds, monkey W: triangles). 
after stimulus onset, but which was not followed by any later wave. Auditory potentials were evoked from a frequency range that was similarly as wide as the range that elicited multiunit responses.

In our sample, $\mathrm{RN}$ neurons had best frequencies that were distributed between 0.16 and $17.75 \mathrm{kHz}$ (median $0.9 \mathrm{kHz}$; Figure 4A) and generally exhibited quite broad tuning curves (median 7.2 octaves; Figure 4B). Responses started between 9 to $17 \mathrm{~ms}$ post-tone onset (median $11 \mathrm{~ms}$; Figure 4C) and lasted, in most cases, for the full duration of the stimulus sound (Figure 4D). We also noted that at 6 sites ( 4 in monkey $\mathrm{E}$ and 2 in monkey $\mathrm{W}$ ) the responses were partially phase locked to the waveform of the tested sounds. The upper limit of phase locking ranged between 0.44 and $1.03 \mathrm{kHz}$ (median $0.9 \mathrm{kHz}$; Figure 4E).

Our experimental approach also allowed us to demonstrate a functional connection from the $\mathrm{RN}$ to the primary auditory cortex. To this end, we applied monopolar electrical stimulation to the RN and performed microelectrode recordings of LFPs and spikes from the auditory cortex. Analysis of 204 stimulation/recording pairs (monkeys $\mathrm{E}$ and $\mathrm{W}$ ) revealed that such stimulation always resulted in an electrically evoked potential in the auditory cortex, which is similar to the representative evoked potentials shown in Figure 5A. The earliest negative trough of the evoked waveform occurred around $34 \mathrm{~ms}$ post-electrical stimulation. This was rapidly followed by a positive peak and a second negative trough, followed by a slow positive deflection ending after about $400 \mathrm{~ms}$ post-stimulation. At a few sites $(n=15,7.4 \%)$ in the auditory cortex, electrical stimulation in the $\mathrm{RN}$ even resulted in an elevated firing of the neurons at a latency of around $50 \mathrm{~ms}$ and a duration of $90 \mathrm{~ms}$ (Figure 5B).

\section{DISCUSSION}

This is the first time that auditory responses have been recorded from the RN of primates, and reveals that groups of neurons that control upper body movement also respond to acoustic signals. The auditory sensitivity observed here is generally in good agreement with that previously observed in chloralose-anesthetized cats (Massion and Albe-Fessard, 1963; Irvine, 1980; Bratus et al., 1981; Shinkarenko, 1984; Shinkarenko et al., 1985). Thus, neurons in the $\mathrm{RN}$ respond with short latencies, to a broad range of tone frequencies, can phase lock their responses to quite high tone frequencies, and respond to the inputs from the two ears, resulting in some selectivity for localizing sound sources. These auditory properties suggest that the RN receives inputs from the non-lemniscal part of the auditory brainstem, even though such projections appear not be present (Massion, 1967; Mylius et al., 2013). A striking difference to the previous studies is the percentage of sites in the $\mathrm{RN}$ at which auditory responses were found. Whereas in all cat studies more than $90 \%$ of neurons were found to respond to clicks or tones, in the monkey RN such responses were only found in around $20 \%$ of the sites. These differences may reflect methodological differences in defining the borders of the RN (e.g., anatomy vs. electrically evoked movements, or anesthetized vs. alert). They may even reflect species differences, such as the importance of pinnae movements for sound source localization. Thus, it is possible that the RN may be under less auditory influence in primates than in lower mammals,

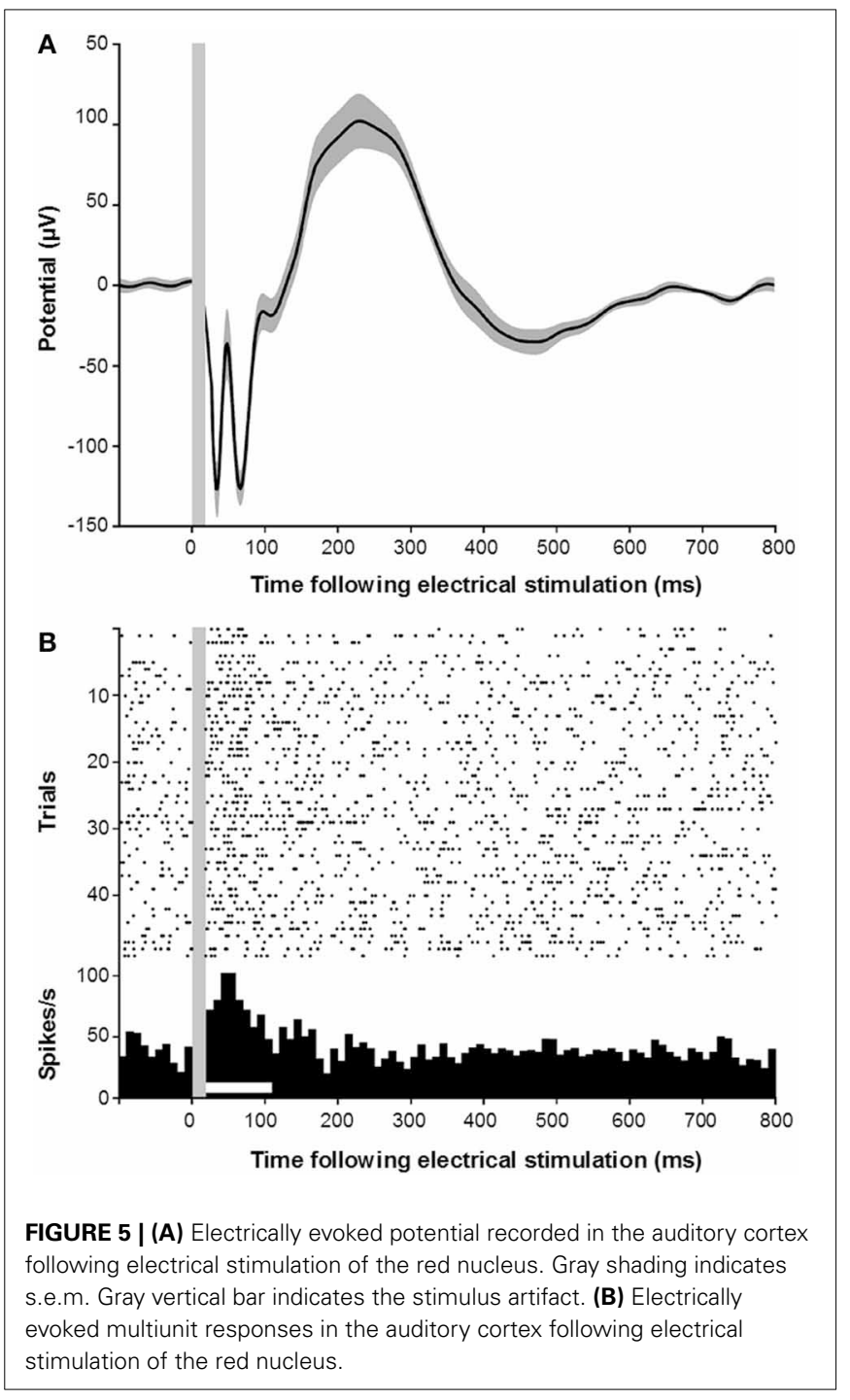

and perhaps reflects a diminished importance of the RN in motor functions, particularly in primates.

The existence of responses to auditory and electrical stimulation in these brainstem nuclei is of considerable use, especially as a landmark when targeting proximal "harder to detect" structures, such as the ventral tegmental area. Our study also suggests a functional connection from the RN to the auditory cortex. Electrical stimulation of the RN-evoked post-synaptic potentials in the auditory cortex, which were suprathreshold in some neurons, thereby causing them to fire action potentials. The latter finding together with the short response latency of $\sim 30 \mathrm{~ms}$, suggests the presence of a projection from the $\mathrm{RN}$ to the auditory cortex that has a slow conduction velocity in the order of $1 \mathrm{~mm} / \mathrm{ms}$ although the existence of this pathway still needs to be anatomically demonstrated.

Our findings corroborate previous accounts that the RN provides a place for audiomotor interactions (Courville, 1968; Martin and Dom, 1970; Shinkarenko, 1984; Shinkarenko et al., 1985). Since the RN appears to receive inputs from a very early stage of the ascending auditory system, our results suggest that 
sounds can modify the motor control exerted by this brain nucleus. Our finding of a functional connection from the RN to the auditory cortex suggests that neuronal activity controlling motor behavior might also affect auditory processing in the auditory system. This pathway might even provide a source for motor-related activity in the auditory cortex (Brosch et al., 2005).

\section{ACKNOWLEDGMENTS}

The authors would like to thank Jörg Stadler for producing the MRI images. Supported by the Deutsche Forschungsgemeinschaft (DFG, SFB 779) and the European Regional Development Fund (ERDF 2007-2013).

\section{REFERENCES}

Bratus, N. V., Shinkarenko, S. A., and Kudriavtseva, I. N. (1981). Electrical responses of the red nucleus to acoustic stimulation. Fiziol. Zh. SSSR Im. I. M. Sechenova 67, 1493-1500.

Brosch, M., and Scheich, H. (2008). Tone-sequence analysis in the auditory cortex of awake macaque monkeys. Exp. Brain Res. 184, 349-361. doi: 10.1007/s00221007-1109-7

Brosch, M., Schulz, A., and Scheich, H. (1999). Processing of sound sequences in macaque auditory cortex: response enhancement. J. Neurophysiol. 82: 1542-1559.

Brosch, M., Selezneva, E., and Scheich, H. (2005). Non-auditory events of a behavioral procedure activate auditory cortex of highly trained monkeys. J. Neurosci. 25, 6797-6806. doi: 10.1523/JNEUROSCI.1571-05.2005

Courville, J. (1968). Connections of the red nucleus with the cerebellum and certain caudal brain stem structures. A review with functional considerations. Rev. Can. Biol. 27, 127.

Gibson, A. R., Houk, J. C., and Kohlerman, N. J. (1985). Magnocellular red nucleus activity during different types of limb movement in the macaque monkey. J. Physiol. 358, 527-549.

Hernandez, R. (1931). Iron content of the brain, its normal and pathological occurrence. Psychiatr. Q. 5, 95-134. doi: 10.1007/BF01574995

Irvine, D. R. (1980). Acoustic input to neurons in feline red nucleus. Brain Res. 200, 169-173. doi: 10.1016/0006-8993(80)91103-8
Martin, G. F., and Dom, R. (1970). Rubrobulbar projections of the opossum (Didelphts virginiana). J.Comp. Neurol. 138, 19-30. doi: 10.1002/cne.901380103 Massion, J. (1967). The mammalian red nucleus. Physiol. Rev. 47, 383-436.

Massion, J., and Albe-Fessard, D. (1963). Dualité des voies sensorielles afférentes contrôlant l'activité du noyau rouge. Electroencephal. Clin. Neurophys. 15, 435-454. doi: 10.1016/0013-4694(63)90065-8

Mylius, J., Brosch, M., Scheich, H., and Budinger, E. (2013). The subcortical auditory structures in the mongolian gerbil: I. Golgi-architecture. J. Comp. Neurol. 521, 1289-1321. doi: 10.1002/cne.23232

Oka, H., and Jinnai, K. (1978). Electrophysiological study of parvocellular red nucleus neurons. Brain Res. 23, 239-246. doi: 10.1016/0006-8993(78)90605-4

Onodera, S., and Hicks, T. P. (2009). A comparative neuroanatomical study of the red nucleus of the cat, macaque and human. PLOS ONE 4:e6623. doi: 10.1371/journal.pone.0006623

Shinkarenko, S. A. (1984). Effect of interaural differences in acoustic stimulation on the neuronal activity of the red nucleus in the cat. Fiziol. Zh. SSSR Im. I. M. Sechenova 70, 291-298.

Shinkarenko, S. A., Bratus, N. V., and Kudriavtseva, I. N. (1985). Neuronal reactions of the red nucleus in cats to acoustic signals. Dokl. Akad. Nauk. SSSR 284, 1506-1509.

Yamaguchi, K., and Goto, N. (2006). Development of the human magnocellular red nucleus: a morphological study. Brain Dev. 7, 431-435. doi: 10.1016/j.braindev.2006.01.001

Conflict of Interest Statement: The authors declare that the research was conducted in the absence of any commercial or financial relationships that could be construed as a potential conflict of interest.

Received: 08 March 2014; accepted: 22 April 2014; published online: 13 May 2014. Citation: Lovell JM, Mylius J, Scheich H and Brosch M (2014) Hearing in action; auditory properties of neurons in the red nucleus of alert primates. Front. Neurosci. 8:105. doi: 10.3389/fnins.2014.00105

This article was submitted to Auditory Cognitive Neuroscience, a section of the journal Frontiers in Neuroscience.

Copyright (c) 2014 Lovell, Mylius, Scheich and Brosch. This is an open-access article distributed under the terms of the Creative Commons Attribution License (CC BY). The use, distribution or reproduction in other forums is permitted, provided the original author(s) or licensor are credited and that the original publication in this journal is cited, in accordance with accepted academic practice. No use, distribution or reproduction is permitted which does not comply with these terms. 